\section{INTERNATIONALLY SUCCESSFUL COMPOSITE}

LuxaCore Z-Dual is DMG UK's logical enhancement of LuxaCore-Dual, their composite for core build-ups, restorative fillings and post cementations, which has been an international success for over ten years.

Thanks to its DMG-patented true nano-technology and the addition of zirconium dioxide, LuxaCore Z-Dual's mechanical properties match the natural tooth more closely than any other material of its category. It has been awarded the Dental Advisor's highest possible ' +++++ ' rating and was named its '2009 Product Award Winner'.

With its radiopaque and pre-silanised LuxaPost, DMG UK offers a glass-fibre reinforced composite post that is perfectly tailored to LuxaCore Z-Dual. Its flexural modulus is very similar to that of the natural dentine thus minimising tension peaks in the post cement - root system and the risk of root fractures.

DMG's dual-curing adhesive system, LuxaBond-

\section{ADVICE ON EVERY ASPECT OF RADIATION}

With dbg, radiation protection is one less thing for you to worry about as a busy dental professional.

With dbg's comprehensive radiation protection service they will supply, inspect, protect, train and advise you on virtually every aspect of dental radiation. The service includes:

- Access to a Radiation Protection Adviser and Medical Physics Expert

- Online Radiation Protection File - a comprehensive file designed to guide you through all your legal requirements

- Quality Assurance Programme

- dbg Helpline - with help and support only a phone call away.

Total Etch, completes this perfect partnership because it is especially suited for adhesive cementation of posts, core build-ups, inlays, onlays, crowns and bridges.

LuxaCore Z-Dual, LuxaPost, LuxaBond-Total Etch: three specialty products that make up a carefully coordinated system for professional

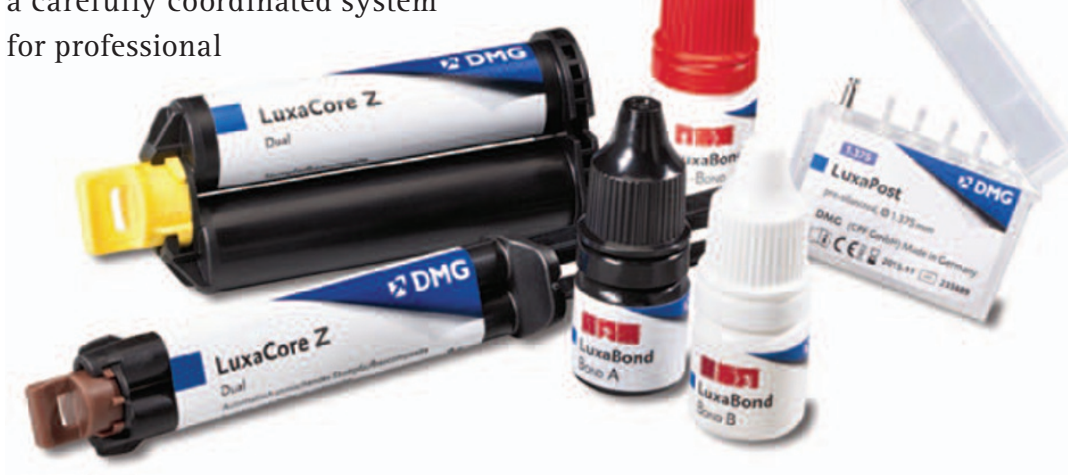

The following services are also included for an additional service cost:

- X-ray Inspection Services

- X-ray Equipment Supply and Installation

- Radiography Protection Courses.

To learn more about dbg's range of radiation protection services, contact the team today. For a limited period dbg is also offering 15\% off their radiation protection service package. Call dbg on 01606861 950 or visit www.thedbg.co.uk.

\section{THE ORIGINAL BULK FILLING MATERIAL}

The range of indications for SDR, the $4 \mathrm{~mm}$ bulk fill composite material, has been extended.

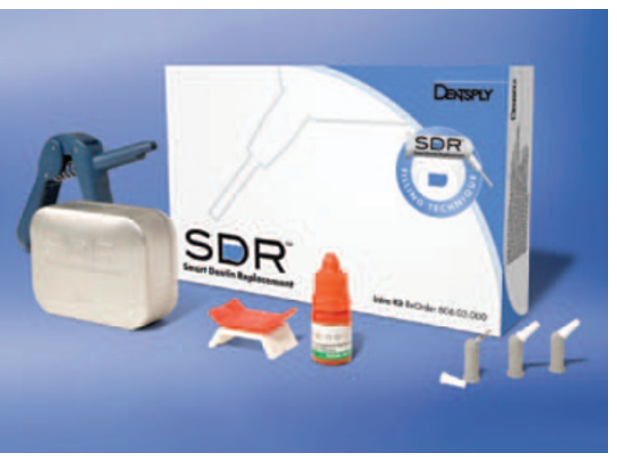

In addition to use as a base in cavity Class I and II direct restorations and a liner under direct restorative materials, SDR has been approved as a fissure sealant, for core build-ups and small Class I restorations in direct occlusal contact without a separate enamel cap.

These extended indications mean that SDR is also ideal for use in paediatric dentistry.

SDR is the original bulk filling material with unique self-levelling properties still unsurpassed when it comes to simplifying the restoration of posterior teeth.

Visit dentsply.co.uk or call 0800072 3313. Earn rewards against purchases at dentsplyrewards. co.uk. Access webinars and products demonstrations and earn CPD at dentsplyacademy.co.uk. post-endodontic restorations.

For further information contact your local dental dealer or DMG Dental Products (UK) Ltd on 01656 789401, emailinfo@ dmg-dental.co.uk or visit www. dmg-dental.com.

\section{METICULOUSLY TESTED $X-R A Y$ MACHINES}

Don't be tempted by the bargain basement price tag. When it comes to X-ray machines, you really do get what you pay for. For meticulously tested, safe and industry approved X-ray equipment, contact Carestream Dental and discover a portfolio of top of the range, CE marked imaging products and solutions.

Try the CS 2100 or CS 2200 $\mathrm{X}$-ray systems, which offer maximum safety, ease of use and reliability. A high frequency generator helps to obtain premier image quality while minimising radiation exposure, delivering up to $25 \%$ less radiation than a standard generator. Compact designs ensure the machines can fit into most practices while sharp, contrasted images offer precise diagnoses with confidence.

Cut-price imports are likely to cut corners elsewhere. With Carestream Dental you not only improve efficiency and treatment but also help to protect your patients and staff.

For more information, contact Carestream Dental on 08001699692 or visit www. carestreamdental.co.uk. 